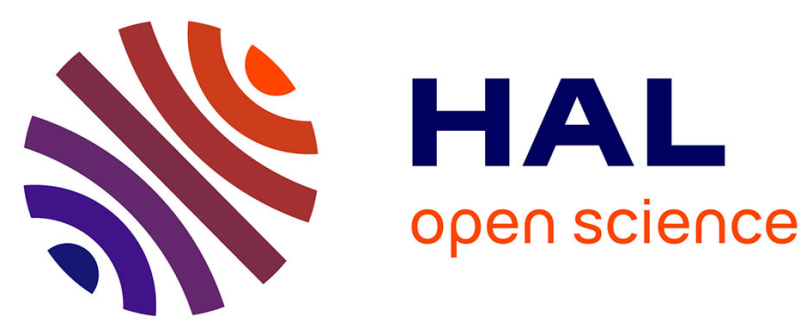

\title{
Opposite effects of ketamine and deep brain stimulation on rat thalamocortical information processing.
}

\author{
Sofya P. Kulikova, Elena A. Tolmacheva, Paul Anderson, Julien Gaudias, \\ Brendan E. Adams, Thomas Zheng, Didier Pinault
}

\section{- To cite this version:}

Sofya P. Kulikova, Elena A. Tolmacheva, Paul Anderson, Julien Gaudias, Brendan E. Adams, et al.. Opposite effects of ketamine and deep brain stimulation on rat thalamocortical information processing.: Ketamine and DBS actions in TC systems. European Journal of Neuroscience, 2012, 36 (10), pp.3407-19. 10.1111/j.1460-9568.2012.08263.x . inserm-00724316

\section{HAL Id: inserm-00724316 https://www.hal.inserm.fr/inserm-00724316}

Submitted on 16 May 2014

HAL is a multi-disciplinary open access archive for the deposit and dissemination of scientific research documents, whether they are published or not. The documents may come from teaching and research institutions in France or abroad, or from public or private research centers.
L'archive ouverte pluridisciplinaire HAL, est destinée au dépôt et à la diffusion de documents scientifiques de niveau recherche, publiés ou non, émanant des établissements d'enseignement et de recherche français ou étrangers, des laboratoires publics ou privés. 
European Journal of Neuroscience

Proposed Journal Section: Neurosystems

\section{Opposite effects of ketamine and deep brain stimulation on rat thalamocortical information processing}

Sofya P Kulikova $(1,2)^{\star}$, Elena A Tolmacheva $(1,2)^{\star \star}$, Paul Anderson $(1,2,3)$, Julien Gaudias $(1,2)^{\star \star \star}$, Brendan E Adams $(1,2)^{\star \star \star \star}$, Thomas Zheng $(1,2,3)$, and Didier Pinault $(1,2)$

(1) INSERM U666, physiopathologie et psychopathologie cognitive de la schizophrénie, Strasbourg, France.

(2) Université de Strasbourg, faculté de médecine, Strasbourg, France.

(3) Department of Medicine, Royal Melbourne Hospital, University of Melbourne, Parkville, VIC, Australia.

Running title: ketamine and DBS actions in TC systems

Number of words: whole ms (5862); Abstract: 247; introduction: 638

Number of figures: 8 , including 2 color figures

Number of tables: 0

Supplementary material: 0

Number of references: 84

Number of pages: 34

Key words : electrophysiology; gamma oscillations; high-frequency electrical stimulation; noise; schizophrenia; sensory-evoked potential.

Correspondence to:

Didier PINAULT, PhD

INSERM U666, Université de Strasbourg

Faculté de Médecine

11, rue Humann

F-67085 Strasbourg Cedex, France

Tel: $+33(0) 368853245$

Fax: +33 (0)3 68853256

Email: pinault@unistra.fr

Current author address:

(*): NEUROSPIN, CEA-Saclay, France

(**): Kharkevich Institute for Information Transmission Problems, Russian Academy of

Sciences, Moscow, Russia

(**): Biozentrum, University of Basel, Switzerland

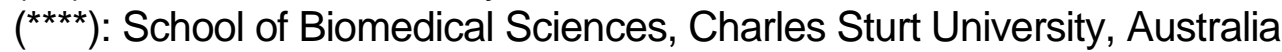




\section{Abstract:}

Sensory and cognitive deficits are common in schizophrenia. They are associated with abnormal brain rhythms, including disturbances in $\gamma$ frequency $(30-80 \mathrm{~Hz}$ ) oscillations (GFO) in cortex-related networks. However, the underlying anatomo-functional mechanisms remain elusive. Clinical and experimental evidence suggest that these deficits result from a hyporegulation of glutamate N-Methyl d-Aspartate receptors (NMDAr). Here we modeled these deficits in rats with ketamine, a non-competitive NMDAr antagonist and a translational psychotomimetic substance at subanesthetic doses. We tested the hypothesis that ketamine-induced sensory deficits involve an impairment of the ability of the thalamocortical (TC) system to discriminate the relevant information from the baseline activity. Furthermore we wanted to assess whether ketamine disrupts synaptic plasticity in TC systems. We conducted multisite network recordings in the rat somatosensory TC system, natural stimulation of the vibrissae and high-frequency electrical stimulation (HFS) of the thalamus. A single systemic injection of ketamine increased the amount of baseline GFO, reduced the amplitude of the sensory-evoked TC response and decreased the power of the sensoryevoked GFO. Furthermore, cortical application of ketamine elicited local and distant increases in baseline GFO. The ketamine effects were transient. Unexpectedly, HFS of the TC pathway had opposite actions. In conclusion, ketamine and thalamic HFS have opposite effects on the ability of the somatosensory TC system to discriminate the sensory-evoked response from the baseline GFO during information processing. Investigating the link between the state and function of the TC system may conceptually be a key strategy to design innovative therapies against neuropsychiatric disorders. 
The anatomofunctional mechanisms of sensory and cognitive deficits in schizophrenia are unknown. These deficits are commonly associated with abnormal brain rhythms, including disturbances in $\gamma$ frequency $(30-80 \mathrm{~Hz}$ ) oscillations (GFO) in corticocortical and thalamocortical (TC) circuits (Bokde et al., 2009; Clinton and Meador-Woodruff, 2004; de Haan W. et al., 2009; Friston, 2002; Herrmann and Demiralp, 2005; Light et al., 2006; Lisman, 2011; Meyer-Lindenberg, 2010; Pinault, 2011; Spencer et al., 2003; Uhlhaas and Singer, 2006). At least four types of GFO should be considered: 1) Spontaneously-occurring (baseline) GFO, which are dominant during desynchronized state of the electroencephalogram (Jasper, 1936; Sheer, 1975); 2) Sensory-evoked GFO, which are phase-locked to the stimulus onset (Pantev et al., 1991; Spencer et al., 2008b); 3) Steadystate GFO during repetitive sensory stimulation (Regan and Spekreijse, 1986); and 4) Cognition or perception-related induced synchronized GFO (Gray et al., 1989; Joliot et al., 1994; Sheer, 1975). Increases and decreases in sensory/task-related GFO (power, frequency, phase synchrony) have been recorded in schizophrenic patients (Herrmann and Demiralp, 2005; Krishnan et al., 2009; Lee et al., 2003; Light et al., 2006; Maharajh et al., 2010; Spencer et al., 2004; Uhlhaas and Singer, 2006). The reported contradictory findings depend on technical aspects, including the location of the cortical recordings, the nature of the symptoms, and the effects of drug treatment. Since sensory and cognitive-related GFO are altered in schizophrenia, one may predict that baseline GFO could somehow modulate them (Hakami et al., 2009). Increased baseline GFO have been recorded in patients during psychotic episodes, including somatic and visual hallucinations (Baldeweg et al., 1998; Becker et al., 2009; Behrendt, 2003; Ffytche, 2008; Spencer et al., 2004), and in early psychosis (Bartha et al., 1997; Theberge et al., 2002). Increases in the amount of baseline GFO are a significant source of excessive network noise in the brain (Baldeweg et al., 1998; Gandal et al., 2011; Hakami et al., 2009; Rolls et al., 2008). Nevertheless, the link between these baseline GFO and the global brain operations and symptoms remains unknown. An increase in GFO could mask sensory-evoked responses and thereby impair sensory information processing (Gandal et al., 2011; Hakami et al., 2009). A postulated mechanism of these impairments is the reduced N-Methyl d-Aspartate receptors (NMDAr) function at glutamatergic synapses on GABAergic interneurons (Coyle, 2006; Moghaddam and Jackson, 2003; Rolls et al., 2008). 
Ketamine is a non-competitive NMDAr antagonist with dose-dependent multipotent properties. It is a drug of abuse with hallucinogenic properties, which strongly impairs neocortical networks (Liao et al., 2011). At a psychotomimetic dose, it acutely induces cognitive and sensory impairments in healthy humans (Boeijinga et al., 2007; Corlett et al., 2007; Hetem et al., 2000; Krystal et al., 1994) and rodents (Chrobak et al., 2008; Ma and Leung, 2007). Thereby, the acute single-dose-ketamine rodent model is promising for understanding the pathogenesis of psychotic states and associated brain network dysfunction (Adell et al., 2011). Indeed, a single injection of ketamine or dizocilpine (or MK801), another non-competitive NMDAr antagonist, also excessively increases the power of baseline GFO (abnormal hypersynchronized GFO) simultaneously in the neocortex and subcortical structures, including the thalamus (Ehrlichman et al., 2009; Hakami et al., 2009; Ma and Leung, 2007; Pinault, 2008).

The current study tested the hypothesis that ketamine-induced sensory deficits are due to an impairment of the ability of the TC system to discriminate the relevant information from the baseline activity. Furthermore, we assessed whether ketamine disrupts synaptic plasticity in TC systems since high-frequency electrical stimulation of the thalamus reliably induces long term potentiation (LTP) of TC synapses (Heynen and Bear, 2001), a potential mechanistic model to estimate the dysfunctional connectivity hypothesis of schizophrenia (Friston, 2002; Stephan et al., 2006). We found that ketamine increases the amount of baseline GFO and decreases the amount of sensory-evoked GFO, and that thalamic highfrequency stimulation has opposite effects. 


\section{MATERIAL AND METHODS}

The major technical challenge was to achieve stable in vivo experimental conditions that closely resemble physiological states for a sufficient time such that the same animal could be recorded under at least two states (eg, control and ketamine) for quantitative and statistical analyses. The freely moving condition is adequate for studying spontaneous activities in correlation with ongoing behavior. However, this condition is unreliable to measure sensory-evoked activities with acceptable precision, especially in the vibrissae system since whiskers rapidly move back and forth during natural behavior. This condition also raises ethical concerns since it would require a relative large number of animals. So, to overcome these difficulties we used two other experimental conditions (light anesthesia and non-anesthetized head-restrained condition) to show whether the observations relative to sensory-evoked activities were specific to a given preparation, each having its own drawbacks and pitfalls. Thereby a system approach was conducted in the rat somatosensory TC system with natural stimulation of the vibrissae and electrical stimulation of the thalamus.

\section{Animals}

Fifty-two adult (3-6-months old) male Wistar rats (280-380 g) were used in accordance with European Union Guidelines (directive 2010/63/EU) and with the approval of the National and Regional Ethics Committee (comité regional d'éthique en matière d'expérimentation animale, Université de Strasbourg). For technical and ethical reasons, and especially in an attempt to reduce the animal number, the experiments were done under 3 experimental conditions: free-moving $(\mathrm{N}=10)$, light anesthesia $(\mathrm{N}=34)$ and acute non-anesthetized head-restrained condition $(\mathrm{N}=8)$. The latter condition was used to validate the findings obtained under light anesthesia, a condition that electrophysiologically corresponds to quiet immobile wakefulness (Pinault, 2003).

\section{Free-moving condition}

The surgical procedures and the experimental conditions are available in a previous report (Pinault, 2008). 


\section{Light anesthesia condition}

The light anesthesia was induced by continuous intravenous injection of the following mixture (quantity given per hour for a 300-g rat): fentanyl $(2 \mu \mathrm{g})$ and glucose (25 mg). Muscle rigidity and tremors were blocked with intravenous administration of D-Tubocurarine chloride $(0.4 \mathrm{mg} / \mathrm{hr})$. The rats were artificially ventilated in the pressure mode $(8-12 \mathrm{~cm}$ $\mathrm{H} 2 \mathrm{O} ; 60 \mathrm{bpm})$ using an O2-enriched gas mixture (50\% air-50\% O2). The rat's rectal temperature was maintained at its physiological level $\left(37-38.3^{\circ} \mathrm{C}\right)$ using a thermoregulated blanket. The electrocorticogram (ECOG) and the heart rate were also under continuous monitoring to maintain a steady depth of light anesthesia either by giving a bolus or adjusting the injection rate of the sedating mixture. The depth of anesthesia was ascertained by the occurrence of slow waves in the ECoG. Recording sessions started 3 hours after state induction. Local anesthetic (lidocaine, 2\%) was infiltrated in all surgical wounds every 2 hours.

Micro-cranioduratomy (diameter $<0.5 \mathrm{~mm}$ ) (Pinault, 2005) was done over the left somatosensory parietal cortex for the insertion of the ACSF-neurobiotin (1\%) filled local field potential (LFP) glass pipette(s) (diameter 5-10 $\mu \mathrm{m}$ ). In the experiments with the thalamic electrical stimulation, an additional opening for the stimulation electrode was done above the somatosensory dorsal thalamus. To prevent the exposed cortical areas from desiccation, surgical sponges soaked with warm artificial cerebrospinal fluid (ACSF) were placed over the openings. Surface monopolar electrocorticogram $(E C O G)$ recordings of the frontoparietal cortex (relative to bregma: posterior $2.3 \mathrm{~mm}$, lateral $5 \mathrm{~mm}$ ) were done with $\mathrm{Ag} / \mathrm{AgCl}$ electrodes (diameter: $150 \mu \mathrm{m}$ ) insulated with teflon and placed in the bone without being in contact with the meninges.

\section{Acute non-anesthetized head-restrained condition}

This condition implied two stages: handling/training and experiment day. Rats were handled and trained individually to stay immobile on a plexiglass plate with their snout in a dark funnel (starting $2 \times 5$ min per day and progressively increasing time up to $2 \times 20 \mathrm{~min}$ ) during at least 1 week. Four days before the experiment day, rats were everyday deprived of water for at least 15 hours. They were trained to stay calm in the funnel as long as possible 
(up to 2x60 min per day) and were rewarded with drops of water. After the last training session they were allowed to drink water up to satiety.

On the experimental day, rats were deeply anesthetized with pentobarbital/ketamine for a standard surgery (see above), which included a micro-cranioduratomy above the target region (parietal somatosensory cortex). A metallic head-restraining system was secured to the cranium with dental cement to preserve painless condition. The rat was laid on the plate with a thermoregulated blanket to maintain it at the physiological temperature $\left(37.5^{\circ} \mathrm{C}\right)$. ECoG electrodes were positioned in the same way as under light anesthesia. Duratomy was done before the insertion of the ACSF-neurobiotin filled LFP pipette(s) and the opening was protected with a surgical sponge soaked with warm ACSF. All open wounds were infiltrated with lidocaine every hour. The transition between anesthetized and non-anesthetized states was made smoother after a subcutaneous injection of a low-dose of diazepam ( $\leqslant 2.5 \mathrm{mg} / \mathrm{kg}$ ). The rat started to recover from anesthesia about 2 hours after induction. The recordings were launched as soon as the rat was awake ( $-3-4$ hours after induction) and was able to drink drops of water, to whisk its vibrissae and to move its limbs and body. The animal was gently handled, helping in maintaining it awake, immobile and calm. Drops of water reward were regularly given. The physiological state of the rat was continuously monitored with rectal temperature, heart rate, breathing, ECoG and electromyogram of neck muscles.

\section{Electrophysiology}

ECoG and LFP were recorded using ultralow noise amplifiers. Signals were processed with a bandpass of $0.1-800 \mathrm{~Hz}$ and digitized at $10 \mathrm{kHz}$. To locate LFP recording site(s), extracellular multiunit labeling (Pinault, 1996) was performed at the end of the recording session (+600 nA, $200 \mathrm{~ms}$ on/-200 ms off, $5 \mathrm{~min}$ ).

Sensory-evoked potentials (see Fig5A) were recorded following contralateral naturallike, mechanical stimulation (back-forth displacement of $2 \mathrm{~mm}$ during $2 \mathrm{~ms} ; 1$ every $5 \mathrm{sec}$ ) of 3-5 vibrissae with a piezo bender actuator. Stimulation intensity was adjusted so that a single stimulus evoked a cortical response $50-60 \%$ of maximal amplitude.

Long-term potentiation of the TC transmission was induced by electrical theta-burst stimulation of the primary somatosensory thalamus (medial ventral posterior nucleus) with a bipolar stimulating semi-micro concentric electrode (diameter: $200 \mu \mathrm{m}$ ). The stimulation 
(theta burst stimulation) consisted of 10 bursts at $5 \mathrm{~Hz}$, with each burst containing 5 pulses at $100 \mathrm{~Hz}$, given four times with a $10 \mathrm{sec}$ interval between each train of 10 bursts. The stimulation settings were described by Heynen and Bear (2001). The location of the stimulation site was confirmed histologically (see Fig7A).

\section{Drugs}

Ketamine (ketamine chlorhydrate [(S)-ketamine and (R)-ketamine]; Imalgene ${ }^{\circledR}$ ) was obtained from Merial (Lyon, France) and MK-801 from Sigma-Aldrich (Saint-Quentin Fallavier, France), pentobarbital from Sanofi (Libourne, France), fentanyl and haldol from Janssen (Boulogne-Billancourt, France). All drugs were dissolved in physiological saline $(\mathrm{NaCl}, 0.9 \%)$. Ketamine was administrated subcutaneously at a single psychotomimetic dose (ketamine $2.5 \mathrm{mg} / \mathrm{kg}$, MK-801 $0.08 \mathrm{mg} / \mathrm{kg}$, unless specified otherwise). In the experiments with local application (see Fig3A), drugs (ketamine, 1mg/ml; MK-801, 0.5 $\mathrm{mg} / \mathrm{ml}$ ) were applied directly to the cortical surface using a soaked surgical sponge placed over the parietal micro-cranioduratomy.

\section{Histology}

At the end of the experiment, the rat was euthanatized by an injection of an overdose of sodium pentobarbital. The brain was subjected to standard histological procedures (Pinault, 2003).

\section{Data analysis}

Data analysis was done using Clampfit 10 (Molecular Devices), Matlab and SciWorks v5 (Datawave Technologies) softwares. Spectral analysis of baseline spontaneously occurring activity was done with Fast Fourier Transformation (FFT) at the resolution of 2.5 $\mathrm{Hz}$ (unless specified otherwise). The power of baseline activity was analyzed in four frequency bands: high-frequency $(81-160 \mathrm{~Hz}), \gamma(30-80 \mathrm{~Hz}$ or GFO), $\beta(16-29 \mathrm{~Hz})$ and slow (1-15 Hz) oscillations. The total power for a given band was computed as the sum of all FFT values in the corresponding frequency range. Correlation in the GFO between different recording sites was addressed by averaged cross-correlation histograms $(\mathrm{N}=7$, successive 
200-ms epochs) and by Pearson's correlation between the values of the $\gamma$ power $(N>100)$ from successive 819-ms epochs recorded at the corresponding sites.

Phase synchronization between two signals was estimated from their mean phase coherence. The phase of a signal was calculated using the Hilbert transform. Then the phase coherence was obtained by projecting the phase differences onto the unit circle in the complex plane and calculating the absolute value of the mean phase difference between two signals (Mormann et al., 2000). The mean phase difference was calculated over 1sepochs (at least 30 epochs per condition).

For sensory-evoked potentials (SEPs) we measured only the amplitude of the first TCrelated short latency component $(10.6 \pm 0.1 \mathrm{~ms}$, negative for LFP, positive for ECoG). The power of the related sensory-evoked GFO was computed from the averaged waveform of 15 post-stimulus 100-ms epochs (see Fig6A). This averaged epoch contains only the sensory-evoked GFO, which occurs at a fixed latency (Tallon-Baudry and Bertrand, 1999). Both baseline and induced GFO are excluded because they are not phase-locked to the stimulus. Spectral power was calculated for saline and ketamine conditions (90 trials each) using the newtimef function from the EEGLAB Matlab toolbox (Delmore and Makeig, 2004). Morlet wavelets were utilized in 80 evenly spaced frequency bands from 20 to $100 \mathrm{~Hz}$, with wavelet cycles ranging from 3 at the lowest frequency to 10 at the highest frequency. Correlation between the amplitude of the SEP and the amount of evoked GFO was given by the Pearson's correlation. All values are presented as means \pm SEM, given either as absolute values or in percentage of change from the averaged values under control condition. Averaging across different rats was performed only with the data presented in the percentage of change. Statistical significance of the observed effects was evaluated with ANOVA, Student t-test and Wilcoxon signed-ranked test (confidence >.95) performed in Systat (v12 Standalone). 


\section{RESULTS}

\section{Correlated increase in baseline GFO between interconnected cortical areas}

To examine the acute impact of a single psychotomimetic dose of ketamine, we conducted multisite high-resolution surface ECoG recordings in free-moving awake rats. Spectral analysis revealed that in all cases $(\mathrm{N}=4)$ a single subcutaneous injection of ketamine $(2.5 \mathrm{mg} / \mathrm{kg})$ significantly and selectively augmented the power of baseline GFO and higher-frequency oscillations (HFO, 81-160 Hz) (Fig1). In addition, we also observed a slight increase in $\beta$ (16-29 Hz) oscillations and no significant change in the power of slower waves $(1-15 \mathrm{~Hz})$. However, at higher doses (ketamine, $5 \mathrm{mg} / \mathrm{kg}$ ), the power of all frequencies was significantly increased (not shown). The observed effects were transient (Fig2B,C) and associated with abnormal behavioral hyperactivity (Hakami et al., 2009; Pinault, 2008). The internal frequency of baseline GFO at maximal power significantly increased by $\sim 5-10 \mathrm{~Hz}$ on average after ketamine injection (Pinault, 2008).

To evaluate the impact of NMDAr antagonists on the spatiotemporal correlation of baseline GFO we assessed the correlation of the power of baseline GFO between highly and weakly interconnected cortical areas before and after ketamine or MK-801 administration. In experiments with bilateral recordings of the parietal cortex (strong anatomical connections via the corpus callosum) drug administration transiently and simultaneously increased GFO power at both recorded sites (Fig2B,C). This effect was characterized by enhanced GFO power in the cross-correlation histogram (Fig2D) and significantly increased the GFO power correlation (Fig2E,F1); in addition, there was no significant difference in the frequency at maximal power (mean $\pm S E M$ : left $\mathrm{cx}: 39.2 \pm 0.4$; right cx: $39.7 \pm 0.5 \mathrm{~Hz}$; t-test: $p>0.05)$. A significant ketamine-induced increase in the correlation of GFO power was likewise observed between the frontal (motor) and parietal (somatosensory) cortices (strong reciprocal anatomical connections; Fig2F2). On the other hand, there was no significant change in $\gamma$ power correlation between the frontal (or parietal) and occipital (visual) cortices, which are anatomically poorly interconnected (Fig2F3); in addition, a significant difference in the frequency at maximal power was recorded between the frontal (or parietal) and occipital cortices (mean \pm SEM: $38.8 \pm 1.1$ and $34.5 \pm 0.8 \mathrm{~Hz}$, respectively; t-test: $p<0.001)$. 
The shift of the central peak of the cross-correlation histogram indicates the time lag between two signals recorded at two different locations (Fig2D). The time lag was $0.12 \pm 0.12 \mathrm{~ms},-1.07 \pm 0.13 \mathrm{~ms}$ and $-5.91 \pm 0.67 \mathrm{~ms}$ between the left and right parietal cortices, the frontal and parietal cortices, and between the motor and visual cortices, respectively. The time lag did not significantly change after a single systemic injection of ketamine or MK801: left-right: $0.00 \pm 0.12 \mathrm{~ms}$ (ttest: $\mathrm{p}>0.05 ; \mathrm{N}=30$ ); frontal-parietal: $-0.83 \pm 0.16 \mathrm{~ms}$ (ttest: $\mathrm{p}=0.247 ; \mathrm{N}=30$ ); frontal-occipital: $-6.10 \pm 0.66 \mathrm{~ms}$ (ttest: $\mathrm{p}=0.312 ; \mathrm{N}=30$ ).

The height of the central peak of the cross-correlogram gives an index of the degree of synchronization of two signals recorded at two distinct locations. GFO had a medium-degree of synchronization between the left and right parietal cortices and between the frontal and parietal cortices $(0.57 \pm 0.02 \mathrm{~ms}$ and $0.55 \pm 0.02 \mathrm{~ms}$, respectively). The synchronization degree between the frontal and occipital cortices is roughly twice lower $(0.25 \pm 0.02 \mathrm{~ms})$. The synchronization degree was not significantly changed after ketamine or MK-801 injection: left-right parietal: $0.59 \pm 0.02$ (ttest: $p>0.05 ; \mathrm{N}=30$ ); frontal-parietal: $0.60 \pm 0.03$ (ttest: $\mathrm{p}=$ $0.208 ; \mathrm{N}=30$ ); frontal-occipital cx: $0.27 \pm 0.02$ (ttest: $\mathrm{p}=0.477 ; \mathrm{N}=30$ ). On the other hand, in all instances the height of the satellite peaks after ketamine injection revealed enhanced strengthening of synchronized GFO during a time window of at least $\pm 100 \mathrm{~ms}$ (Fig2D). This merely revealed simultaneous increase in $\gamma$ power.

The phase synchronization of brain oscillations is thought to be an important dynamic network mechanism involved in various integration processes (Varela et al., 2001). Phase synchronization of GFO is reduced in patients with schizophrenia (Herrmann and Demiralp, 2005; Lee et al., 2003; Maharajh et al., 2010; Spencer et al., 2003; Uhlhaas and Singer, 2006). Therefore, we wanted to assess the impact of ketamine on the mean phase coherence of GFO simultaneously recorded between the left and right parietal cortices. In all free-moving rats $(\mathrm{N}=4)$ no significant change was observed after a single injection of ketamine or MK-801 relative to saline injection. Indeed, whatever the condition the phase coherence was quite stable over time with an average value of $0.60 \pm 0.01(\mathrm{~N}>30$ 1-s epochs per condition).

Thus, ketamine significantly increases correlation of the GFO power between strongly but not between poorly anatomically interconnected cortical areas, supporting the commonly 
accepted hypothesis of the existence of multiple independent generators of baseline GFO, at least in the cerebral cortex.

\section{Local modulation of GFO}

If the multiple generator hypothesis is true and knowing that non-competitive NMDAr antagonists can act directly into brain networks (Hunt et al., 2010), local application of ketamine (or MK-801) onto a restricted area of the neocortex should create a focus of GFO hyperactivity (Fig3A). To test this hypothesis intracortical LFP were simultaneously recorded in the supra- and/or infragranular layers of the parietal somatosensory cortex along with a surface ECoG $\sim 2 \mathrm{~mm}$ away. At the end of the experiment extracellular labeling (Pinault, 1996) was performed for histological verification of the location of the LFP recording sites. These experiments were conducted under light anesthesia $(\mathrm{N}=9)$ and validated under the acute non-anesthetized head-restrained condition $(\mathrm{N}=3)$. In all instances, local application of

ketamine (or MK-801, results not shown) created a focus of baseline GFO hyperactivity: a rapid ( $\sim 5-8$ min after application) and significant increase in GFO power just below the site of application without any immediate impact in the adjacent ECoG (Fig3B1,B2,C). Washing ketamine away led to a significant decrease of the GFO power in the intracortical LFP recordings, while a slight increase was observed in the ECoG, which was likely due to ketamine diffusion. On the other hand, subcutaneous injection of ketamine simultaneously produced a fast and significant increase in the GFO power at all recording sites (Fig3B1,B2,C). Under both the head-restrained and the free-moving conditions, a systemic injection of ketamine augmented the behavioral activity (limb movements). Taken together, these findings indicate that non-competitive NMDAr antagonists have powerful effects on network GFO generators.

\section{Distant modulation of GFO}

The ability of local application of NMDAr antagonists to alter local ongoing GFO raised the question whether local application of such antagonists can modulate ongoing GFO in distant connected structures. In an attempt to answer this question we performed paired ECoG recordings of the left (site of application) and right parietal cortices along with the right occipital cortex under light anesthesia (Fig4A); a LFP electrode was also introduced in the 
cortex that was submitted to local drug application to monitor the local effects. In all instances (9 ketamine conditions paired with ACSF conditions, 5 rats) ketamine increased the amount of spontaneously occurring GFO in both the intracortical LFP and the adjacent left ECoG (Student's t-test: $p<0.01$ ). One-way ANOVA revealed a significant difference in ketamine-induced changes in $\gamma$ power in the three recording sites $(F(2,218)=301.2$, $p<0.0001$; Fig4B). Bonferroni's post-hoc test showed significant differences between the right parietal and occipital electrodes $(p<0.001)$. The effect in the right parietal ECoG was modest, relatively to the left ECoG (close to the ketamine application site). No significant increase in $\gamma$ power was recorded in the right occipital cortex, which is weakly or not connected with the left parietal cortex. In addition no significant change in both the power coherence and the phase coherence was observed between the left and right parietal cortices after local ketamine application. These results suggest that local ketamine application can increase the amount of GFO in distant and interconnected (here via the corpus callosum) cortical networks.

\section{Increased baseline GFO and decreased TC response}

In any system, both the amount of its background activity and the amplitude of the systemic response to a single external stimulation are powerful indicators of its state and functionality. Here we used these parameters to evaluate the impact of ketamine on the somatosensory TC system. The level of background activity was estimated from the power of baseline GFO recorded with both the intracortical LFP pipette and the surface ECoG (Fig5A). Sensory-evoked potentials were induced by mechanical stimulation of 3-5 vibrissae. We measured the amplitude of the TC response, which corresponds to the first component of the SEP (Heynen and Bear, 2001; Simons et al., 2007), the positive peak in the ECoG (latency: $10.8 \pm 0.2 \mathrm{~ms}, \mathrm{~N}=64$ ) and the negative peak in the intracortical LFP $(10.5 \pm 0.2 \mathrm{~ms}, \mathrm{~N}=64)$. Under light anesthesia (4 rats) or non-anesthetized head-restrained (3 rats) condition (Fig5B,C), a single subcutaneous injection of ketamine significantly altered both the state and functioning of TC circuits, simultaneously increasing the amount of baseline cortical GFO and decreasing the amplitude of the SEP. Thus ketamine decreases the sensory signal-to- $\gamma$-noise ratio. The ketamine-induced effects were basically similar in both the surface ECoG and the related intracortical LFP (Fig5B,C). This result was 
expected since, under our experimental conditions, the surface ECoG activities mirror those recorded in the subjacent intracortical networks (Hakami et al., 2009).

\section{Decreased sensory-evoked GFO}

We then investigated the effect of ketamine on sensory-evoked $\gamma$ activity, defined as a burst of additional GFO that occurs at a fixed latency after the stimulus (Tallon-Baudry and Bertrand, 1999) (Fig6A; see methods for details). Time-frequency analysis revealed that ketamine increased the amount of baseline GFO (Fig6A, before stimulus given at $0 \mathrm{~ms}$ ) and decreased the amount of evoked GFO (Fig6A, during the post-stimulus 100-ms epoch). Correlation analysis revealed a strong positive correlation between the absolute amplitude of the sensory-evoked TC responses and the power of evoked GFO (Fig6B; average Pearson correlation coefficient (mean \pm SEM): ECoG: $0.56 \pm 0.03$, LFP: $0.75 \pm 0.04, N=7$ rats: 4 rats under light anesthesia and 3 under non-anesthetized head-restrained condition). This led to two predictions: i) evoked GFO present a "true" sensory-related component of the SEP, i.e. carrying stimulus-related information; ii) since ketamine increases the baseline $\gamma$ power and decreases the amplitude of the TC response (Fig5B,C, Fig6A,C), it should also decrease the related amount of evoked GFO. Indeed, our experiments revealed that ketamine led to a significant decrease in the power of sensory-evoked GFO (Fig6C).

\section{Ketamine disrupts thalamocortical LTP}

Network and synaptic activities are intimately linked. Synapses are responsible for dynamic processes that regulate neuronal responsiveness and plasticity. Sensory-evoked TC responses include NMDAr-dependent component (Heynen and Bear, 2001;Salt et al., 1988). So, if ketamine decreases the amplitude of the sensory-evoked TC response, it is expected to reduce the LTP, at least of the TC neurotransmission (Heynen and Bear, 2001). Therefore, we tested under light anesthesia the impact of ketamine on LTP in the adult somatosensory TC system. The experimental design was identical to the one from Fig5A with the addition of a stimulating electrode inserted into the primary somatosensory thalamus for delivering HFS (Fig7A). LTP, assessed with the sensory-evoked TC response, was reliably induced in a sustainable fashion for at least 5 hours following thalamic stimulation (Fig7B). The first thalamic electrical stimulation was applied only when no 
significant change in the amplitude of the TC response was recorded during at least one hour. Moreover, in some instances $(\mathrm{N}=6)$, LTP was induced by repetitive sensory stimulation alone (Fig7C). In an attempt to assess the possibility that potentiation was also in part due to the electrode insertion into the thalamus, we conducted a series of experiments without the intrathalamic stimulation electrode. An increase in the TC response amplitude was observed in 2 out of 5 experiments (not shown). Therefore, we cannot exclude the possibility that potentiation could also be "naturally" induced by repetitive sensory stimulation alone, in agreement with previous observations (Megevand et al., 2009). As expected, administration of ketamine (or MK-801) transiently disrupted LTP of TC transmission and in parallel increased the amount of baseline GFO (Fig7C,E,F).

Surprisingly and of importance, under ketamine-free condition thalamic HFS that tetanized TC inputs to get LTP also produced a consistent decrease in the amount of baseline GFO (Fig7D). In other words, thalamic HFS increased the signal-to- $\gamma$-noise ratio, an effect that was opposite to the ketamine-induced effect observed under the same experimental conditions. 


\section{DISCUSSION}

The novelty of the present study is that NMDAr hypofunction impairs the ability of the somatosensory TC system to discriminate the sensory-evoked activity from the background GFO. Indeed, a single injection of a psychotomimetic dose of ketamine or MK-801 simultaneously increases the power of baseline GFO and decreases both the amplitude of the sensory-evoked TC response and the power of its related GFO. These new findings are in resonance with clinical neurophysiological observations from patients with schizophrenia (Herrmann and Demiralp, 2005; Krishnan et al., 2009; Lee et al., 2003; Light et al., 2006; Maharajh et al., 2010; Spencer et al., 2003; Spencer et al., 2004; Spencer et al., 2008a; Uhlhaas and Singer, 2006) and support both the hypothesis of dysfunctional TC circuits in schizophrenia (Clinton and Meador-Woodruff, 2004; Ferrarelli and Tononi 2010; Friston, 2002; Lisman, 2011; Meyer-Lindenberg, 2010; Pinault, 2011; Stephan et al., 2006), and the hypothesis of a reduction of the cortical signal-to-noise ratio in schizophrenia (Gandal et al., 2011; Rolls et al., 2008).

Surprisingly, repetitive stimulation of the TC pathway (either by thalamic HFS or by vibrissae stimulation) provokes effects that are opposite to those induced by ketamine. Taken together these findings may conceptually be of paramount importance for the advancement of our understanding of the pathogenesis of psychotic states and provide novel clues for the preclinical development of innovative therapeutic strategies of schizophrenia.

In the present study, the ketamine-induced electrophysiological effects recorded under light anesthesia were not specific to this experimental condition since they were validated in the non-anesthetized condition (also see Hakami et al., 2009). Whether or not the baseline GFO, recorded under normal or ketamine condition, have a functional role is a true question (Bernasconi et al., 2011) that merits further studies in our quest to understand the pathophysiology of schizophrenia (Rolls et al., 2008). The present study shows that ketamine-induced simultaneous multisite increases in baseline GFO are not associated with a significant change in phase coherence, at least between strongly interconnected cortical areas, but are consistently associated with an increase in $\gamma$ power coherence. This is an argument in favor of the hypothesis that increased synchronization in baseline GFO between distant cortical areas does not reflect integration processes. It is worth pointing out 
that a significant augmentation in the amount of baseline GFO has also been recorded during and in between prominent slow waves after injection of a narcotic or anesthetic agent (Hakami et al., 2009), including after a single anesthetizing dose of ketamine (Maksimow et al., 2006).

The signal-to- $\gamma$-noise ratio (defined here as a ratio between the power of the sensoryevoked and baseline GFOs) in neural circuits is considered as a suitable neurophysiological marker to evaluate their dysfunction, both in patients with schizophrenia and in animal models for schizophrenia (Ehrlichman et al., 2009; Gandal et al., 2011; Rolls et al., 2008; Winterer et al., 2000). A recent clinical review (Gandal et al., 2011) suggests that abnormalities in GFO are ubiquitous in schizophrenia. They involve both an augmentation of baseline cortical GFO and a reduction of stimulus-evoked $\gamma$ response, thereby affecting the signal-to- $\gamma$-noise ratio during cortical information processing. Here, we provide the first direct experimental evidence for the impairment of the signal-to- $\gamma$-noise ratio in a sensory TC system in an acute NMDAr-dependent model for the pathogenesis of psychoses. Therefore, our findings, together with the facts that a psychotomimetic dose of ketamine increases the $\gamma$ power in humans (Hong et al., 2010) and that ketamine induces schizophrenia-like symptoms (Gunduz-Bruce, 2009; Hetem et al., 2000; Krystal et al., 1994), stress the translational potential of GFO as a biomarker for positive symptoms (Hakami et al., 2009; Pinault, 2008). Moreover, continuous and stereotyped GFO were proposed to be responsible for clinical positive symptoms (Llinas et al., 1999).

The present findings support the hypothesis of the existence of multiple independent cortical generators of GFO. Indeed, local cortical application of ketamine or MK-801 proved that such generators can be modulated independently. It is further shown that local cortical application of ketamine can modulate the amount of baseline GFO in distant connected (at least via the corpus callosum) structures. This effect was not observed between distant cortical areas having weak or no anatomical connections. Further investigation is needed to understand the underlying mechanisms. The origin, size and intrinsic connectivity of GFO generators are beyond the scope of the present study (Whittington et al., 2011).

In the present work we show that the power of sensory-evoked GFO is highly correlated with the amplitude of the TC response and therefore should be considered as a signal component that carries stimulus-driven information, which might be gated by increased 
baseline cortical GFO (Wang et al., 2010). Furthermore, the observed opposite effects of NMDAr antagonists on the amount of spontaneous and evoked GFO suggest that they are generated by different mechanisms, pointing out that evoked GFO may not be just a mere sensory-evoked augmentation of baseline GFO. Our results are in agreement with a model based on a neural network simulation, in which spontaneous and stimulus-driven GFO exist independently and can resonate under the control of synaptic plasticity of TC connections (Paik and Glaser, 2010).

Since baseline GFO were similarly increased after systemic or local application of NMDAr antagonists, we predict that the underlying mechanisms were identical. Possible mechanisms of the acute effects of non-competitive NMDAr antagonists were discussed elsewhere (Gunduz-Bruce, 2009; Hakami et al., 2009; Moghaddam and Jackson, 2003). Briefly, assuming that the observed ketamine-induced effects are mainly due to the specific blockade of NMDAr, NMDAr hypofunction is thought to lead to increased glutamatergic transmission, which would subsequently activate non-NMDAr (AMPA and kainate receptors, Fuchs et al., 2007). In spite of a possible imbalance in the NMDA/AMPA ratio, we should also keep in mind experimental findings showing that ketamine as well acts at $A C h, 5 H T 2$, D2 and GABA receptors (Flood and Krasowski, 2000; Irifune et al., 2000;Kapur and Seeman, 2002).

Presynaptic mechanisms might also be involved in the ketamine-induced behavioral and neurophysiological effects. Indeed, in the rat pretreatment with metabotropic glutamate2/3 receptors agonists significantly attenuated PCP-induced behavioral and cognitive effects and reduced synaptic glutamate release (Moghaddam and Adams, 1998). Furthermore, pretreatment with such agonist (LY379268) significantly attenuated the ketamine-induced increase in the power of baseline GFO (Jones et al., 2011).

Also, one should be prudent when comparing results and inferring mechanisms from studies using different doses of NMDAr antagonists and/or several types of models (Bubenikova-Valesova et al., 2008). Indeed, here a single low-dose of ketamine (2.5 mg/kg) or MK-801 (0.08 mg/kg) increased principally GFO. At higher doses ketamine impacted also slower $(\alpha, \theta, \delta)$ waves as observed by other teams (Ehrlichman et al., 2009; Hong et al., 2010; Palenicek et al., 2011; Tsuda et al., 2007). Such electrophysiological abnormalities are observed in chronic schizophrenia (Ferrarelli et al., 2007; Herrmann and Demiralp, 2005; 
Moran and Hong, 2011; Uhlhaas and Singer, 2006). Therefore it is tempting to suggest that the low-dose ketamine rat model, used in the present study, models more hyperfrontality, which can be observed in first-episode schizophrenia (Callicott et al., 2000), than the hypofrontality that is inherent to chronic schizophrenia. So, the single low-dose ketamine model may be appropriate to model the pathogenesis of acute psychotic states, a model that is translatable in humans (Corlett et al., 2007; Hetem et al., 2000; Krystal et al., 1994).

What are the neuronal mechanisms underlying the observed transient ketamine-induced disruption of the sensory signal-to- $\gamma$-noise ratio in TC networks? Recent experimental (Bikbaev and Manahan-Vaughan, 2008) and computational (Paik and Glaser, 2010) studies suggest a strong link between baseline GFO and synaptic plasticity. Also, disruption of the synaptic functioning represents an important mechanistic model for the dysfunctional connectivity hypothesis of schizophrenia (Friston, 2002; Stephan et al., 2006). Here, ketamine- or MK-801-induced disruption of the LTP of TC synapses is in agreement with these two hypotheses, although somehow predictable since LTP depends in part on NMDAr activation (Heynen and Bear, 2001). It should however be emphasized that ketamine and MK-801 transiently decreased the potentiated TC response but did not stop the development of the LTP. Moreover, this effect paralleled the impact of NMDAr antagonists on baseline GFO (Pinault, 2008). Now, whether or not ketamine prevents LTP induction is a question that merits further investigation.

Surprisingly, we observed that, while LTP was developing after thalamic electrical tetanization, the power of baseline GFO was decreasing in the related cortical area. This means that thalamic HFS (here in the primary somatosensory nucleus) has effects opposite to the NMDAr antagonist-induced effects. Moreover, experimental findings support the concept that thalamic HFS has certain pro-sensory/cognitive properties possibly linked to LTP mechanisms (Mair and Hembrook, 2008; Shirvalkar et al., 2006). Our findings revealed that the signal-to- $\gamma$-noise ratio can be considered as a key characteristic that determines both the state and the function of a given circuit (Fig8). However, the physiological relevance to a behavioral state of our stimulation paradigm, used under acute conditions, remains an issue requiring further studies.

The mechanisms underlying the effects of thalamic HFS on the basal GFO and TC response are unknown. Whatever the anatomical target, it is generally recognized that HFS 
primarily excites axons and rapidly inhibits, via depolarization block, cell bodies and dendrites (Deniau et al., 2010; Kringelbach et al., 2007). So we predict that intrathalamic HFS should at least excite TC and corticothalamic pathways and afferent/passing axons, subsequently generating immediate and long-lasting multiple effects, one of these being changes in the synaptic plasticity of TC synapses.

In conclusion, we have demonstrated that investigating GFO abnormalities and the signal-to-noise ratio in the somatosensory TC system is relevant to understand the pathophysiology of (first-episode) schizophrenia. Deciphering the neural mechanisms underlying disruption of information processing in models of mental disorders and in patients will contribute to understanding their pathophysiology and may therefore conceptually help creating novel therapeutic avenues. 


\section{Acknowledgements:}

Anne-Sophie Bouillot provided technical assistance (Inserm Apprenticeship, Professional Licence, University Joseph Fourier, Grenoble, France). S.K. was a fellow of the Trinational Neurex Joint Master in Neuroscience (Basel-Strasbourg-Freiburg) and of Inserm; E.T. was supported by a Inserm Postdoctoral Fellowship; T.Z. had a Eiffel Doctorat Fellowship of Excellence (2007-2008); P.A. received an Australian Rotary Health lan Scott PhD scholarship and a 2012 Australian Endeavour Research Fellowship Award; J.G. was a Master student of Université de Strasbourg; B.A. was supported by a Neurex Fellowship. The present work was funded by Inserm, Université de Strasbourg, la Fondation pour la Recherche Médicale and Neurex.

\section{Abbreviations:}

ACSF, artificial cerebrospinal fluid; ECoG, electrocorticogram; FFT, Fast Fourier Transformation; GFO, $\gamma$ frequency $(30-80 \mathrm{~Hz})$ oscillations; HFS, high-frequency stimulation; LFP, local field potential; LTP, long-term potentiation; NMDAr , N-Methyl d-Aspartate receptor; SEP, sensory-evoked potential; TC, thalamocortical.

\section{Competing interests:}

SK, ET, PA, JG, BEA, TZ, and DP report no biomedical financial interests or potential conflicts of interest.

\section{Author Contributions:}

S.K. completed the experiments (LTP, evoked potential, $\gamma$ oscillations and local application under anesthesia and non-anesthetized head-restrained conditions), analyzed the data and wrote the manuscript;

E.T. completed the experiments (LTP under anesthesia) and analyzed the data; P.A. completed the experiments (impact of local ketamine application in distant anatomically connected structures), analyzed the data and contributed to the writing.

J.G. completed the experiments (local application under anesthesia) and analyzed the data; B.A. completed the experiments (evoked potential and $\gamma$ oscillations under anesthesia) and analyzed the data;

T.Z. contributed to the experiments (LTP and local application under anesthesia); 
D.P. Designed the experiments, completed the experiments, analyzed the data and wrote the paper. 
Reference List

Adell,A., Jimenez-Sanchez,L., Lopez-Gil,X. \& Romon,T. (2012) Is the Acute NMDA Receptor Hypofunction a Valid Model of Schizophrenia? Schizophr.Bull., 38, 9-14.

Baldeweg,T., Spence,S., Hirsch,S.R. \& Gruzelier,J. (1998) Gamma-band electroencephalographic oscillations in a patient with somatic hallucinations. Lancet, 352, 620-621.

Bartha,R., Williamson,P.C., Drost,D.J., Malla,A., Carr,T.J., Cortese,L., Canaran,G., Rylett,R.J. \& Neufeld,R.W. (1997) Measurement of glutamate and glutamine in the medial prefrontal cortex of never-treated schizophrenic patients and healthy controls by proton magnetic resonance spectroscopy. Arch.Gen.Psychiatry, 54, 959-965.

Becker,C., Gramann,K., Muller,H.J. \& Elliott,M.A. (2009) Electrophysiological correlates of flicker-induced color hallucinations. Conscious. Cogn., 18, 266-276.

Behrendt,R.P. (2003) Hallucinations: synchronisation of thalamocortical gamma oscillations underconstrained by sensory input. Conscious. Cogn., 12, 413-451.

Bernasconi,F., De,L.M., Tzovara,A., Manuel,A.L., Murray,M.M. \& Spierer,L. (2011) Noise in Brain Activity Engenders Perception and Influences Discrimination Sensitivity. J. Neurosci., 31, 17971-17981.

Bikbaev,A. \& Manahan-Vaughan,D. (2008) Relationship of hippocampal theta and gamma oscillations to potentiation of synaptic transmission. Front. Neurosci., 2, 56-63.

Boeijinga,P.H., Soufflet,L., Santoro,F. \& Luthringer,R. (2007) Ketamine effects on CNS responses assessed with MEG/EEG in a passive auditory sensory-gating paradigm: an attempt for modelling some symptoms of psychosis in man. J.Psychopharmacol., 21, 321-337.

Bokde,A.L., Ewers,M. \& Hampel,H. (2009) Assessing neuronal networks: understanding Alzheimer's disease. Prog.Neurobiol., 89, 125-133.

Bubenikova-Valesova,V., Horacek,J., Vrajova,M. \& Hoschl,C. (2008) Models of schizophrenia in humans and animals based on inhibition of NMDA receptors. Neurosci.Biobehav.Rev., 32, 1014-1023.

Callicott,J.H., Bertolino,A., Mattay,V.S., Langheim,F.J., Duyn,J., Coppola,R., Goldberg,T.E. \& Weinberger,D.R. (2000) Physiological dysfunction of the dorsolateral prefrontal cortex in schizophrenia revisited. Cereb.Cortex, 10, 1078-1092. 
Chrobak,J.J., Hinman,J.R. \& Sabolek,H.R. (2008) Revealing past memories: proactive interference and ketamine-induced memory deficits. J.Neurosci., 28, 4512-4520.

Clinton,S.M. \& Meador-Woodruff,J.H. (2004) Thalamic dysfunction in schizophrenia: neurochemical, neuropathological, and in vivo imaging abnormalities. Schizophr.Res., 69, 237-253.

Corlett,P.R., Honey,G.D. \& Fletcher,P.C. (2007) From prediction error to psychosis: ketamine as a pharmacological model of delusions. J. Psychopharmacol., 21, 238-252.

Coyle,J.T. (2006) Glutamate and schizophrenia: beyond the dopamine hypothesis. Cell Mol.Neurobiol., 26, 365-384.

de Haan W., Pijnenburg,Y.A., Strijers,R.L., van der,M.Y., van der Flier,W.M., Scheltens,P. \& Stam,C.J. (2009) Functional neural network analysis in frontotemporal dementia and Alzheimer's disease using EEG and graph theory. BMC.Neurosci., 10, 101.

Delorme, A. \& Makeig, S. (2004) EEGLAB: an open source toolbox for analysis of single trial EEG dynamics including independent component analysis. J. Neurosci. Methods, 134, 9-21.

Deniau,J.M., Degos,B., Bosch,C. \& Maurice,N. (2010) Deep brain stimulation mechanisms: beyond the concept of local functional inhibition. Eur.J. Neurosci, 32, 1080-1091.

Ehrlichman,R.S., Gandal,M.J., Maxwell,C.R., Lazarewicz,M.T., Finkel,L.H., Contreras,D., Turetsky,B.I. \& Siegel,S.J. (2009) N-methyl-d-aspartic acid receptor antagonist-induced frequency oscillations in mice recreate pattern of electrophysiological deficits in schizophrenia. Neuroscience, 158, 705-712.

Ferrarelli,F., Huber,R., Peterson,M.J., Massimini,M., Murphy,M., Riedner,B.A., Watson,A., Bria,P. \& Tononi,G. (2007) Reduced sleep spindle activity in schizophrenia patients. Am.J.Psychiatry, 164, 483-492.

Ferrarelli,F. \& Tononi,G. (2010) The Thalamic Reticular Nucleus and Schizophrenia. Schizophr.Bull., 37, 306-315.

Ffytche,D.H. (2008) The hodology of hallucinations. Cortex, 44, 1067-1083.

Flood,P. \& Krasowski,M.D. (2000) Intravenous anesthetics differentially modulate ligandgated ion channels. Anesthesiology, 92, 1418-1425.

Friston,K.J. (2002) Dysfunctional connectivity in schizophrenia. World Psychiatry, 1, 66-71. 
Fuchs,E.C., Zivkovic,A.R., Cunningham,M.O., Middleton,S., Lebeau,F.E., Bannerman, D.M., Rozov,A., Whittington, M.A., Traub,R.D., Rawlins,J.N. \& Monyer,H. (2007) Recruitment of parvalbumin-positive interneurons determines hippocampal function and associated behavior. Neuron, 53, 591-604.

Gandal,M.J., Edgar,J.C., Klook,K. \& Siegel,S.J. (2012) Gamma synchrony: Towards a translational biomarker for the treatment-resistant symptoms of schizophrenia. Neuropharmacology, 62, 1504-1518.

Gray,C.M., Konig,P., Engel,A.K. \& Singer,W. (1989) Oscillatory responses in cat visual cortex exhibit inter-columnar synchronization which reflects global stimulus properties. Nature, 338, 334-337.

Gunduz-Bruce,H. (2009) The acute effects of NMDA antagonism: from the rodent to the human brain. Brain Res.Rev., 60, 279-286.

Hakami,T., Jones,N.C., Tolmacheva,E.A., Gaudias,J., Chaumont,J., Salzberg,M., O'Brien,T.J. \& Pinault,D. (2009) NMDA receptor hypofunction leads to generalized and persistent aberrant gamma oscillations independent of hyperlocomotion and the state of consciousness. PLoS.One., 4, e6755.

Herrmann,C.S. \& Demiralp,T. (2005) Human EEG gamma oscillations in neuropsychiatric disorders. Clin.Neurophysiol., 116, 2719-2733.

Hetem,L.A., Danion,J.M., Diemunsch,P. \& Brandt,C. (2000) Effect of a subanesthetic dose of ketamine on memory and conscious awareness in healthy volunteers. Psychopharmacology (Berl), 152, 283-288.

Heynen,A.J. \& Bear,M.F. (2001) Long-term potentiation of thalamocortical transmission in the adult visual cortex in vivo. J.Neurosci., 21, 9801-9813.

Hong,L.E., Summerfelt,A., Buchanan,R.W., O'Donnell,P., Thaker,G.K., Weiler,M.A. \& Lahti,A.C. (2010) Gamma and delta neural oscillations and association with clinical symptoms under subanesthetic ketamine. Neuropsychopharmacology, 35, 632-640.

Hunt,M.J., Falinska,M. \& Kasicki,S. (2010) Local injection of MK801 modifies oscillatory activity in the nucleus accumbens in awake rats. J.Psychopharmacol., 24, 931-941.

Irifune,M., Sato,T., Kamata,Y., Nishikawa,T., Dohi,T. \& Kawahara,M. (2000) Evidence for $\operatorname{GABA}(\mathrm{A})$ receptor agonistic properties of ketamine: convulsive and anesthetic behavioral models in mice. Anesth.Analg., 91, 230-236. 
Jasper,H.H. (1936) Cortical excitatory state and variability in human brain rhythms. Science, 83, 259-260.

Joliot,M., Ribary,U. \& Llinas,R. (1994) Human oscillatory brain activity near $40 \mathrm{~Hz}$ coexists with cognitive temporal binding. Proc. Natl. Acad. Sci. U.S.A., 91, 11748-11751.

Jones,N.C., Reddy,M., Anderson,P., Salzberg,M.R., O'Brien,T.J. \& Pinault,D. (2012) Acute administration of typical and atypical antipsychotics reduces EEG gamma power, but only the preclinical compound LY379268 reduces the ketamine-induced rise in gamma power. Int.J. Neuropsychopharmacol., 15, 657-668.

Kapur,S. \& Seeman,P. (2002) NMDA receptor antagonists ketamine and PCP have direct effects on the dopamine $\mathrm{D}(2)$ and serotonin $5-\mathrm{HT}(2)$ receptors-implications for models of schizophrenia. Mol.Psychiatry, 7, 837-844.

Kringelbach,M.L., Jenkinson,N., Owen,S.L. \& Aziz,T.Z. (2007) Translational principles of deep brain stimulation. Nat.Rev.Neurosci., 8, 623-635.

Krishnan,G.P., Hetrick,W.P., Brenner,C.A., Shekhar,A., Steffen,A.N. \& O'Donnell,B.F. (2009) Steady state and induced auditory gamma deficits in schizophrenia. Neuroimage., 47, 1711-1719.

Krystal,J.H., $\quad$ Karper,L.P., $\quad$ Seibyl,J.P., $\quad$ Freeman,G.K., $\quad$ Delaney,R., $\quad$ Bremner,J.D., Heninger,G.R., Bowers,M.B., Jr. \& Charney,D.S. (1994) Subanesthetic effects of the noncompetitive NMDA antagonist, ketamine, in humans. Psychotomimetic, perceptual, cognitive, and neuroendocrine responses. Arch.Gen.Psychiatry, 51, 199-214.

Lee,K.H., Williams,L.M., Haig,A. \& Gordon,E. (2003) "Gamma (40 Hz) phase synchronicity" and symptom dimensions in schizophrenia. Cognit.Neuropsychiatry, 8, 57-71.

Liao,Y., Tang,J., Corlett,P.R., Wang,X., Yang,M., Chen,H., Liu,T., Chen,X., Hao,W. \& Fletcher,P.C. (2011) Reduced dorsal prefrontal gray matter after chronic ketamine use. Biol.Psychiatry, 69, 42-48.

Light,G.A., Hsu,J.L., Hsieh,M.H., Meyer-Gomes,K., Sprock,J., Swerdlow,N.R. \& Braff,D.L. (2006) Gamma band oscillations reveal neural network cortical coherence dysfunction in schizophrenia patients. Biol.Psychiatry, 60, 1231-1240.

Lisman,J. (2011) Excitation, inhibition, local oscillations, or large-scale loops: what causes the symptoms of schizophrenia? Curr. Opin. Neurobiol., 22, 537-544. 
Llinas,R.R., Ribary,U., Jeanmonod,D., Kronberg,E. \& Mitra,P.P. (1999) Thalamocortical dysrhythmia: A neurological and neuropsychiatric syndrome characterized by magnetoencephalography. Proc. Natl. Acad. Sci. U.S.A, 96, 15222-15227.

Ma,J. \& Leung,L.S. (2007) The supramammillo-septal-hippocampal pathway mediates sensorimotor gating impairment and hyperlocomotion induced by MK-801 and ketamine in rats. Psychopharmacology (Berl), 191, 961-974.

Maharajh,K., Teale,P., Rojas,D.C. \& Reite,M.L. (2010) Fluctuation of gamma-band phase synchronization within the auditory cortex in schizophrenia. Clin. Neurophysiol., 121, 542-548.

Mair,R.G. \& Hembrook,J.R. (2008) Memory enhancement with event-related stimulation of the rostral intralaminar thalamic nuclei. J. Neurosci., 28, 14293-14300.

Maksimow,A., Särkelä,M., Långsjö,J.W., Salmi,E., Kaisti,K.K., Yli-Hankala, A., Hinkka-YliSalomäki,S., Scheinin,H. \& Jääskeläinen,S.K. (2006) Increase in high frequency EEG activity explains the poor performance of EEG spectral entropy monitor during Sketamine anesthesia. Clin. Neurophysiol., 117, 1660-1668.

Megevand,P., Troncoso,E., Quairiaux,C., Muller,D., Michel,C.M. \& Kiss,J.Z. (2009) Longterm plasticity in mouse sensorimotor circuits after rhythmic whisker stimulation. J.Neurosci., 29, 5326-5335.

Meyer-Lindenberg,A. (2010) From maps to mechanisms through neuroimaging of schizophrenia. Nature, 468, 194-202.

Moghaddam,B. \& Adams,B.W. (1998) Reversal of phencyclidine effects by a group II metabotropic glutamate receptor agonist in rats. Science, 281, 1349-1352.

Moghaddam,B. \& Jackson,M.E. (2003) Glutamatergic animal models of schizophrenia. Ann.N.Y.Acad.Sci., 1003, 131-137.

Moran,L.V. \& Hong,L.E. (2011) High vs low frequency neural oscillations in schizophrenia. Schizophr.Bull., 37, 659-663.

Mormann,F., Lehnertz,K., David,P. \& Elger, C.E. (2000). Mean phase coherence as a measure for phase synchronization and its application to the EEG of epilepsy patients. Physica D, 144, 358-369.

Paik,S.B. \& Glaser,D.A. (2010) Synaptic plasticity controls sensory responses through frequency-dependent gamma oscillation resonance. PLoS.Comput.Biol., 6. 
Palenicek,T., Fujakova,M., Brunovsky,M., Balikova,M., Horacek,J., Gorman,I., Tyls,F., Tislerova,B., Sos,P., Bubenikova-Valesova,V., Hoschl,C. \& Krajca,V. (2011) Electroencephalographic spectral and coherence analysis of ketamine in rats: correlation with behavioral effects and pharmacokinetics. Neuropsychobiology, 63, 202218.

Pantev,C., Makeig,S., Hoke,M., Galambos,R., Hampson,S. \& Gallen,C. (1991) Human auditory evoked gamma-band magnetic fields. Proc.Natl.Acad.Sci.U.S.A., 88, 89969000.

Pinault,D. (1996) A novel single-cell staining procedure performed in vivo under electrophysiological control: morpho-functional features of juxtacellularly labeled thalamic cells and other central neurons with biocytin or Neurobiotin. J. Neurosci. Methods, 65, 113-136.

Pinault,D. (2003) Cellular interactions in the rat somatosensory thalamocortical system during normal and epileptic 5-9 Hz oscillations. J. Physiol., 552, 881-905.

Pinault,D. (2005) A new stabilizing craniotomy-duratomy technique for single-cell anatomoelectrophysiological exploration of living intact brain networks. J. Neurosci. Methods, 141, 231-242.

Pinault,D. (2008) N-methyl d-aspartate receptor antagonists ketamine and MK-801 induce wake-related aberrant gamma oscillations in the rat neocortex. Biol.Psychiatry, 63, 730735.

Pinault,D. (2011) Dysfunctional Thalamus-Related Networks in Schizophrenia. Schizophr.Bull., 37, 238-243.

Regan,D. \& Spekreijse,H. (1986) Evoked potentials in vision research 1961-86. Vision Res., 26, 1461-1480.

Rolls,E.T., Loh,M., Deco,G. \& Winterer,G. (2008) Computational models of schizophrenia and dopamine modulation in the prefrontal cortex. Nat.Rev.Neurosci., 9, 696-709.

Salt,T.E., Wilson,D.G. \& Prasad,S.K. (1988) Antagonism of N-methylaspartate and synaptic responses of neurones in the rat ventrobasal thalamus by ketamine and MK-801. Br. J. Pharmacol., 94, 443-448.

Sheer,D.E. (1975) Behavior and brain electrical activity Plenum Press, New York and London. 
Shirvalkar,P., Seth,M., Schiff,N.D. \& Herrera,D.G. (2006) Cognitive enhancement with central thalamic electrical stimulation. Proc.Natl.Acad.Sci.U.S.A, 103, 17007-17012.

Simons,D.J., Carvell,G.E., Kyriazi,H.T. \& Bruno,R.M. (2007) Thalamocortical conduction times and stimulus-evoked responses in the rat whisker-to-barrel system. J.Neurophysiol., 98, 2842-2847.

Spencer,K.M., $\quad$ Nestor,P.G., $\quad$ Niznikiewicz,M.A., $\quad$ Salisbury,D.F., $\quad$ Shenton,M.E. ～\& McCarley,R.W. (2003) Abnormal neural synchrony in schizophrenia. J. Neurosci., 23, 7407-7411.

Spencer,K.M., Nestor,P.G., Perlmutter,R., Niznikiewicz,M.A., Klump,M.C., Frumin,M., Shenton,M.E. \& McCarley,R.W. (2004) Neural synchrony indexes disordered perception and cognition in schizophrenia. Proc. Natl. Acad. Sci.U.S.A., 101, 17288-17293.

Spencer,K.M., Niznikiewicz,M.A., Shenton,M.E. \& McCarley,R.W. (2008a) Sensory-evoked gamma oscillations in chronic schizophrenia. Biol.Psychiatry, 63, 744-747.

Spencer,K.M., Salisbury,D.F., Shenton,M.E. \& McCarley,R.W. (2008b) Gamma-band auditory steady-state responses are impaired in first episode psychosis. Biol.Psychiatry, 64, 369-375.

Stephan,K.E., Baldeweg,T. \& Friston,K.J. (2006) Synaptic plasticity and dysconnection in schizophrenia. Biol.Psychiatry, 59, 929-939.

Tallon-Baudry,C. \& Bertrand,O. (1999) Oscillatory gamma activity in humans and its role in object representation. Trends Cogn. Sci., 3, 151-162.

Theberge,J., Bartha,R., Drost,D.J., Menon,R.S., Malla,A., Takhar,J., Neufeld,R.W., Rogers,J., Pavlosky,W., Schaefer,B., Densmore,M., Al-Semaan,Y. \& Williamson,P.C. (2002) Glutamate and glutamine measured with 4.0 T proton MRS in never-treated patients with schizophrenia and healthy volunteers. Am.J.Psychiatry, 159, 1944-1946.

Tsuda,N., Hayashi,K., Hagihira,S. \& Sawa,T. (2007) Ketamine, an NMDA-antagonist, increases the oscillatory frequencies of alpha-peaks on the electroencephalographic power spectrum. Acta. Anaesthesiol.Scand., 51, 472-481.

Uhlhaas,P.J. \& Singer,W. (2006) Neural synchrony in brain disorders: relevance for cognitive dysfunctions and pathophysiology. Neuron, 52, 155-168.

Varela,F., Lachaux,J.P., Rodriguez,E. \& Martinerie, J. (2001) The brainweb: phase synchronization and largescale integration. Nature Rev. Neurosci. 2, 229-239. 
Wang,H.P., Spencer,D., Fellous,J.M. \& Sejnowski,T.J. (2010) Synchrony of thalamocortical inputs maximizes cortical reliability. Science, 328, 106-109.

Whittington,M.A., Cunningham,M.O., LeBeau,F.E., Racca,C. \& Traub,R.D. (2011) Multiple origins of the cortical gamma rhythm. Dev. Neurobiol., 71, 92-106.

Winterer,G., Ziller,M., Dorn,H., Frick,K., Mulert,C., Wuebben,Y., Herrmann,W.M. \& Coppola,R. (2000) Schizophrenia: reduced signal-to-noise ratio and impaired phaselocking during information processing. Clin. Neurophysiol., 111, 837-849. 


\section{FIGURE LEGENDS}

Figure 1. Low-dose ketamine increases the amount of baseline $\gamma$ and highfrequency oscillations. All recordings and data analyses are from awake free-moving rats. A) Typical 500-ms episodes of frontal ECoG under control and ketamine $(2.5 \mathrm{mg} / \mathrm{kg})$ or MK$801(0.08 \mathrm{mg} / \mathrm{kg})$ conditions. Asterisks indicate bursts of low-amplitude high-frequency oscillations (HFO, 81-160 Hz). B) A typical expanded trace showing four $\gamma$ waves flanked by two HFO bursts (asterisks). C) The chart shows the power spectrum (resolution of $2.4 \mathrm{~Hz}$; each point being the average of more than 2000 epochs of $410 \mathrm{~ms}$ ) of baseline ECoG under control and ketamine (C1) or MK-801 (C2) conditions. The histograms are from two typical experiments (control/ketamine and control/MK-801) and shows the total power at 4 frequency bands: slow (1-15 Hz), $\beta$ (16-29 Hz), $\gamma$ (30-80 Hz or GFO) and HFO. The values are means $\left( \pm\right.$ SEM). A single low-dose of ketamine or MK-801 significantly $\left({ }^{*}, p<0.001\right.$, $t-$ test) increases the power of $\gamma$ and HFO, less the amount of $\beta$ oscillations, and has no significant (ns) effect on the power of slow oscillations.

Figure 2. Ketamine increases baseline GFO power correlation between interconnected cortical areas. A) Dorsal view of the cranium showing the location of the recording cortical electrodes. Two series of experiments were done in awake rats: i) bilateral recording of the parietal cortex (Lpar, Rpar); ii) triple recording of the frontal (fr), parietal (par) and occipital (occ) cortices (ref for reference). The thick and dotted double-ended arrows indicate strong and weaker anatomical connections between two cortical areas, respectively. B) Typical 1-s episodes of Lpar and Rpar recordings under control condition, 15 and 70 min after ketamine (keta) injection. C) Kinetics of the keta effects on the power of baseline GFO over Lpar and Rpar (arrows indicate injection time). D) Average crosscorrelation histograms (7 successive 200-ms epochs) of baseline activity recorded over the Lpar and Rpar cortices (control condition) and $\sim 15$ min after keta injection. E) Keta increases the degree of linear relationship (Pearson's correlation) between successive paired Lpar-Rpar GFO power values ( $>100$, 819-ms epochs). F1-F3) Pearson correlation 
coefficient significantly increases between interconnected cortical areas after ketamine injection ( ${ }^{*}$,Wilcoxon signed-ranked test, $p<0.05$; ns for non-significant).

Figure 3. Local application of ketamine modulates local network GFO. A) Experimental design showing the location of ECoG and LFPs (LFPup, LFPdown) recording sites. Pictures of a micro-cranioduratomy (left), showing intact neuro-glio-vascular networks, and of extracellular labeling of the LFP recording sites (right). B-C) Baseline GFO power (in $\%$ from baseline) after local ketamine and ACSF (washing for control) applications followed by a systemic ketamine (vehicle or veh for control) administration. These experiments were made under light anesthesia (B1-B2, 9 rats) and awake head-restrained condition (C, 3 rats). Local applications and systemic administration of ketamine were done in the same rat under the same experimental conditions. In (C) the vertical grey bars indicate the periods during which the recordings were contaminated by electromyographic activities (due to handling, drop of water, local application or systemic injection). B2) Local application significantly ( ${ }^{*}$, t-test, $\left.p<0.01\right)$ increases the GFO power just below the application site but does not immediately impact on the ECoG, while systemic injection has significant ( ${ }^{*}$, t-test, $\mathrm{p}<0.01$ ) effect at all recording sites. Each column (black and white for local and systemic administrations, respectively) is an average ( \pm SEM) of 220 values from 273 ms-epochs. The vehicle is saline. bg : bregma; ns : non-significant.

Figure 4. Local application of ketamine can modulate distant network GFO. A) Experimental design showing the location of the ECoG electrodes. The experimental conditions (under light anesthesia) of local application of ketamine or ACSF are similar to those shown in figure 3A. B) Average change in the power of baseline GFO recorded simultaneously in the left and right parietal cortices (Lpar and Rpar) and in the right occipital cortex (Rocc). For each condition (ACSF or ketamine) a drop of the corresponding solution was applied every 15 min (arrows). The average values ( \pm SEM) are from 9 points $\times 5$ rats.

Figure 5. Ketamine increases the amount of baseline GFO and decreases the amplitude of the sensory-evoked TC response. A) Experimental design showing the simplified 3-neuron circuit involving the neocortex, the GABAergic thalamic reticular nucleus 
(TRN) and the thalamus (Th). Natural-like mechanical stimulation (stim) of the vibrissae is provided by a piezo bender actuator. Baseline cortical activity and sensory-evoked potentials (SEP) are recorded simultaneously with the surface electrocorticogram (ECoG) electrode and intracortical (layer IVN, verified histologically) local field potential (LFP) micropipette. The inset shows a typical SEP (average of 15 sweeps) recorded in the ECoG dependent component (positive in ECoG, negative in LFP, double dotted lines) is measured. B1,2) Each pair of charts presents the temporal dynamics of the SEP amplitude (lower chart) and the power of baseline (basal) GFO (basal $\gamma$; upper chart, total power in the 30-80 $\mathrm{Hz}$ range on the $200 \mathrm{~ms}$ intervals before the corresponding sensory stimulations) in two typical experiments, one under light anesthesia (B1), the other under head-restrained condition (B2). Ketamine (keta, $2.5 \mathrm{mg} / \mathrm{kg}$, subcutaneous) is administrated $80 \mathrm{~min}$ after the beginning of the experiment (arrow). Each point is an average ( \pm SEM) of 15 values. C1,2) Quantitative and statistical analysis under light anesthesia (4 rats, C1) and under nonanesthetized head-restrained condition (3 rats, C2) reveal significant $\left({ }^{*}, \mathrm{t}\right.$-test, $\left.\mathrm{p}<0.0001\right)$ simultaneous changes in both the power of basal GFO (basal $\gamma$; increase) and the sensoryevoked TC amplitude (ev TC resp; decrease) (white columns for control condition, black columns for ketamine condition). Each column in the histograms is the average ( \pm SEM) of at least 45 values collected during 20-30 min under each condition. Values for ketamine condition are taken from $\sim 10-15$ min after injection.

Figure 6. Ketamine decreases the amount of sensory-evoked GFO. A) Averaged intracortical LFP (in black) and ECoG (in green) SEPs recorded (filtered in the $\gamma$ range) in a non-anesthetized head-restrained rat under control (cont) and ketamine conditions. Below is shown the related time-frequency graph of the ECoG for each condition (90 LFP SEP trials, stimulus given at $\mathbf{O m s}$ ). B) The amplitude of the sensory-evoked TC component is positively correlated with the power of sensory-evoked GFO (Pearson correlation coefficient $(P c c)=0.69, p<0.001, N=30$ values from 3 rats, head-restrained condition). C) Quantitative and statistical analysis ( ${ }^{*}$, t-test, $\left.p<0.0001\right)$ show that ketamine administration increases the power of basal GFO and decreases the power of evoked GFO. The averaged power of the basal GFO is measured during the 100-ms epoch before the sensory stimulation (s stim; from at least 45 trials, 3 rats). The power of the sensory-evoked GFO is directly measured 
from the average SEP (12 values from the post-stimulus 100-ms epoch from 3 rats per condition; see methods).

Figure 7. NMDAr antagonists transiently disrupt thalamocortical LTP. A) Histological control of the location of the stimulation site (asterisk) in the medial ventral posterior nucleus (VPm). B) Thalamic theta burst stimulation (2 applications: TBS1 \& TBS2) increases the SEP amplitude in the LFP; each point is the average ( \pm SEM) of 4 ratsx15 values. C) Ketamine transiently disrupts sensory-induced LTP. Top: changes in the baseline GFO power; bottom: changes in the SEP amplitude before and after ketamine. Each point is an average of 15 values $\times 4$ rats ( \pm SEM). D) TBS decreases the baseline $\gamma$ power; each point is the average ( \pm SEM) of 4 rats $\times 25$ values from 250 -ms epochs. E-F) Ketamine or MK-801 disrupts TBS-induced LTP. Each column is the average ( \pm SEM) of $6 \times 15$ successive values. Experimental conditions: control (cont), $\sim 20$ min after TBS1, $\sim 40$ min after TBS2, 15 min after saline (veh), 15 and 60 min after ketamine (keta, D) or 15 and 120 min after MK-801 (MK, E). Stars indicate significant difference relative to the previous condition (t-test, $p<0.01$ ), all values are in $\%$ from the baseline.

Figure 8. Opposite effects of ketamine and high-frequency deep brain stimulation (hfDBS) on TC information processing. This synopsis sums up the principal findings of the present study: Ketamine increases the amount of baseline GFO and decreases the amplitude of the sensory-evoked TC response; thalamic hfDBS has an opposite effect and wherefore could probably reverse ketamine-induced effects. 
A

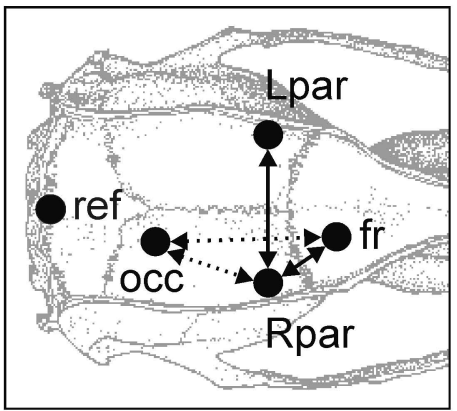

c
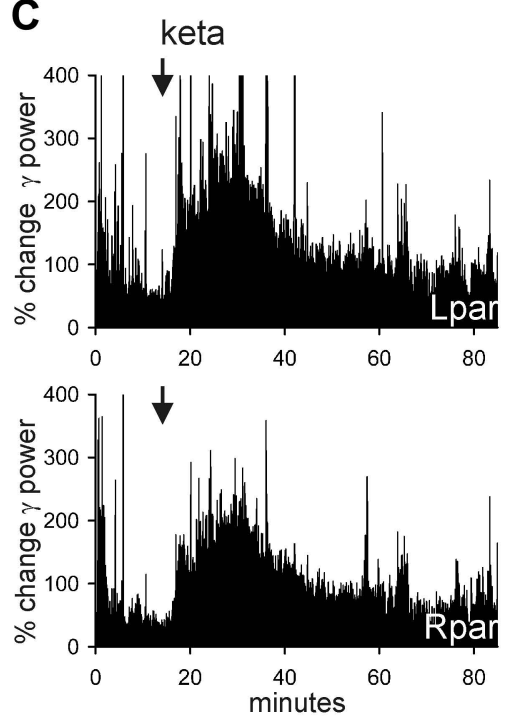

F1

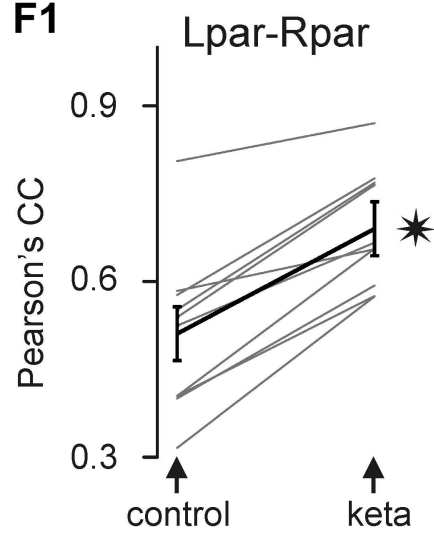

B

control

keta +15 min

keta +70 min

Lpar

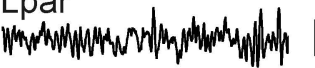

Rpar $50 \mu \mathrm{V}$

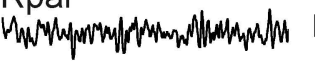

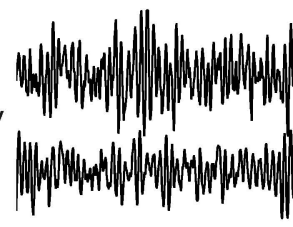

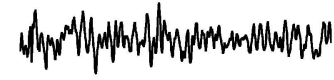

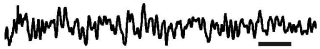
$100 \mathrm{~ms}$

$$
\text { Lpar } \rightarrow \text { Rpar }
$$

control
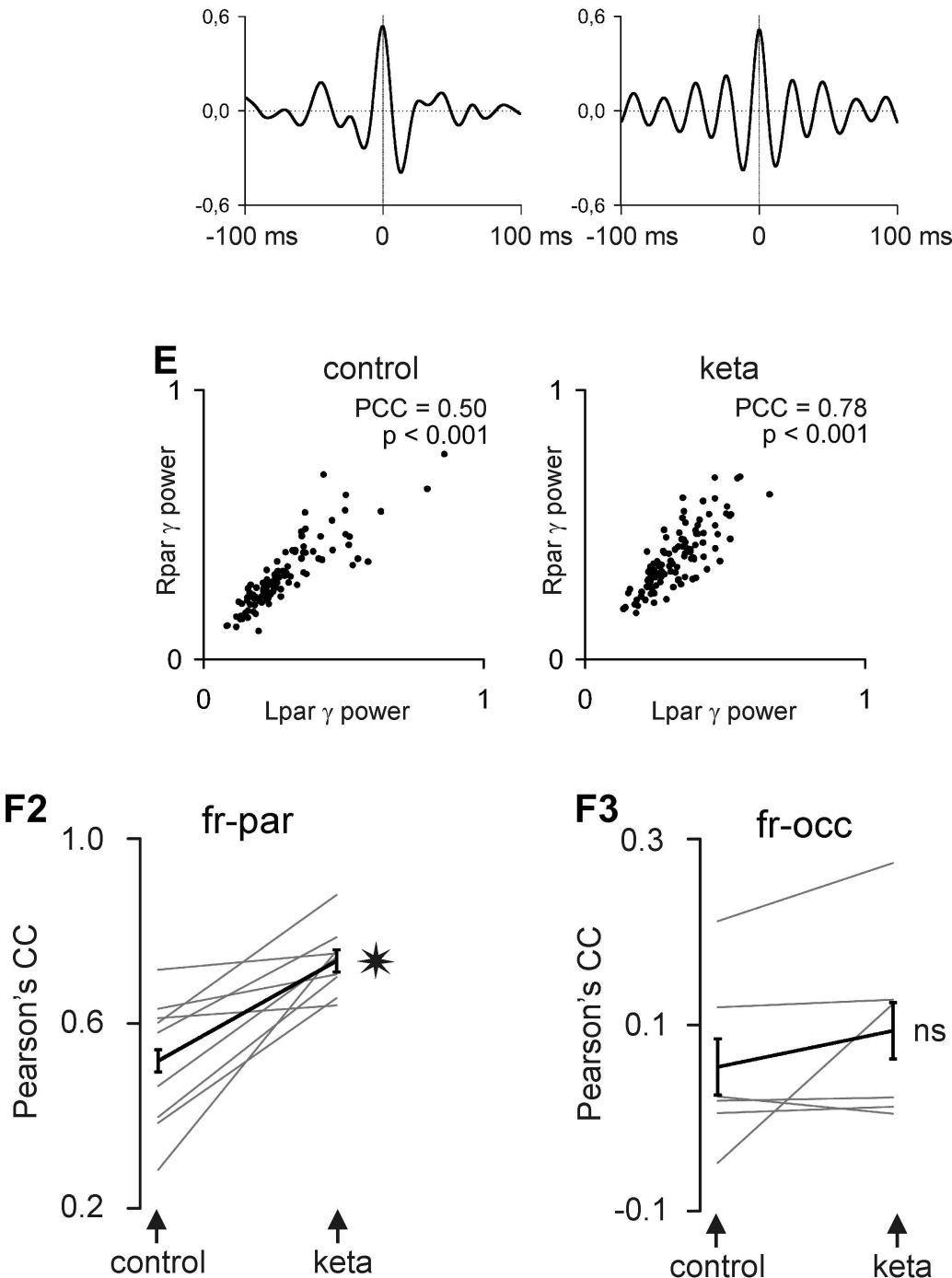

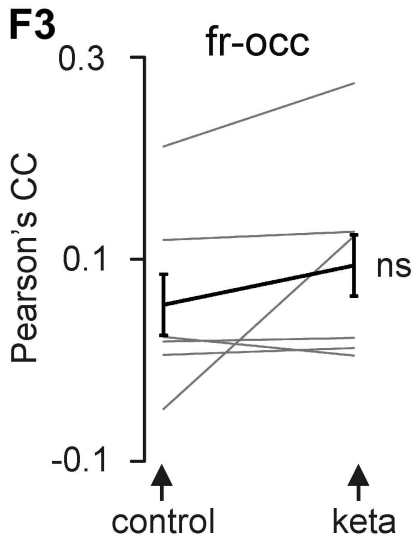




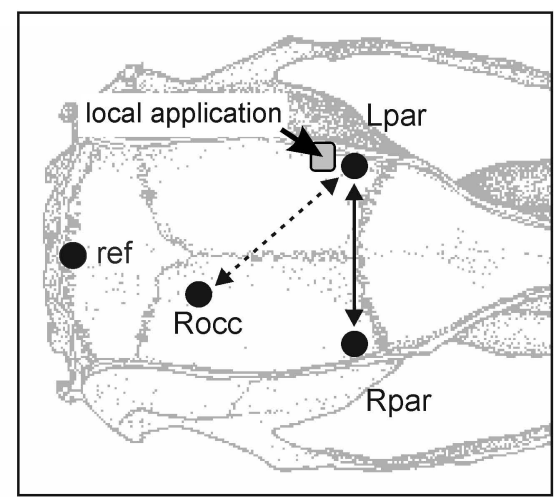

B

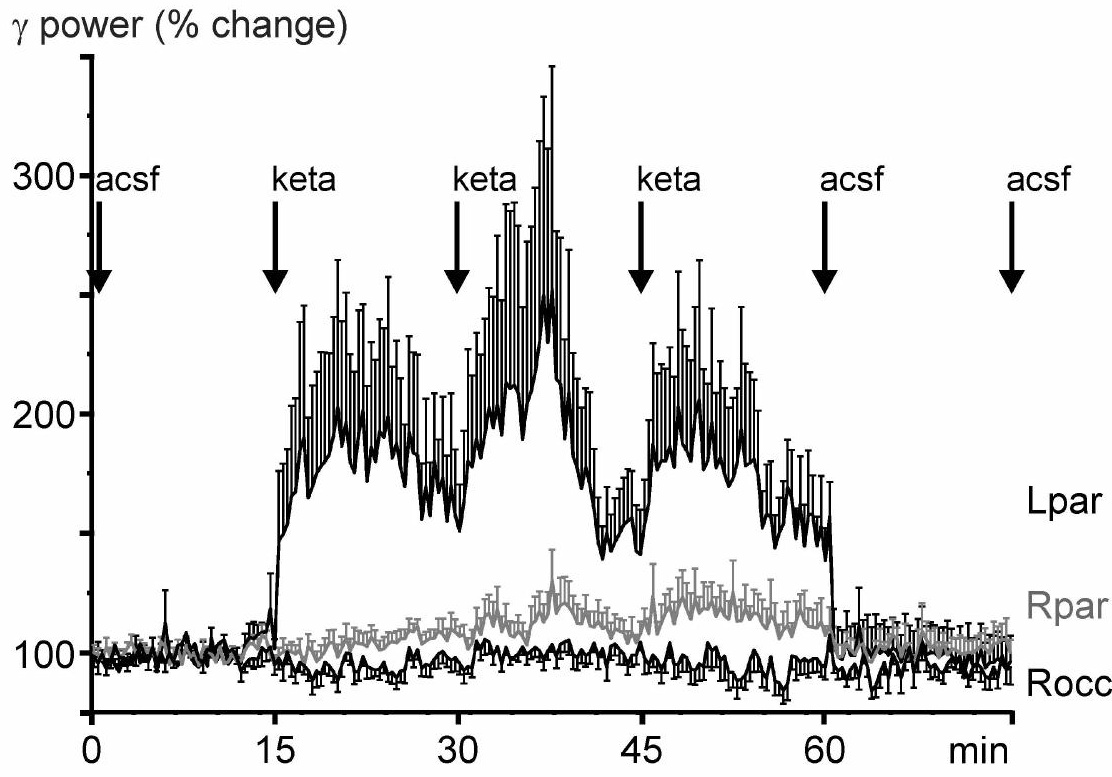




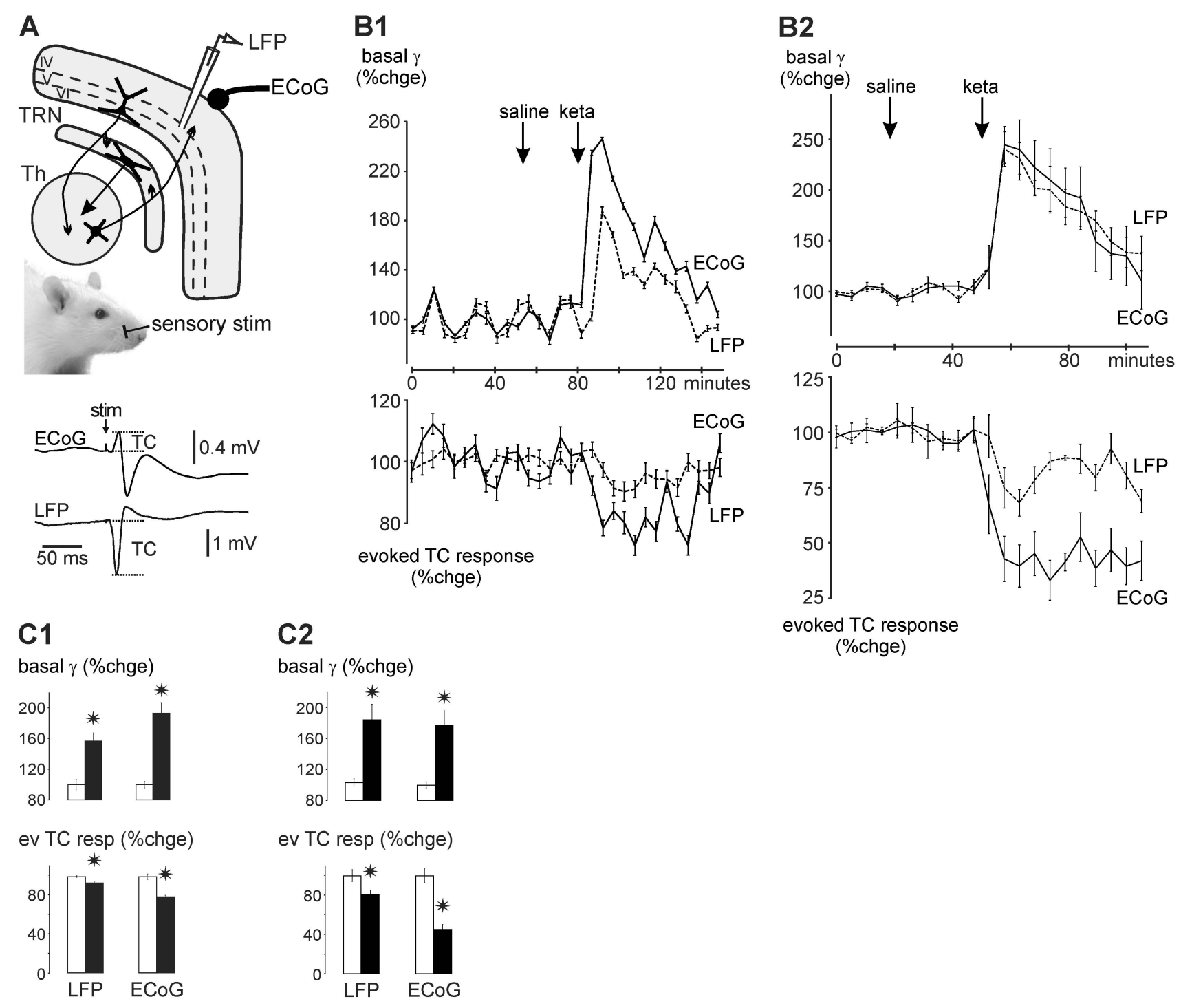


control
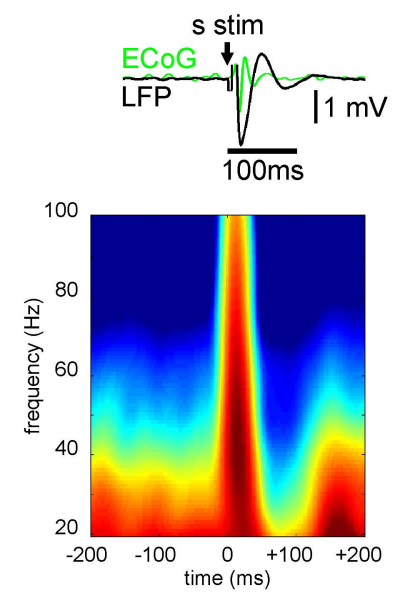

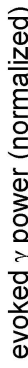

B

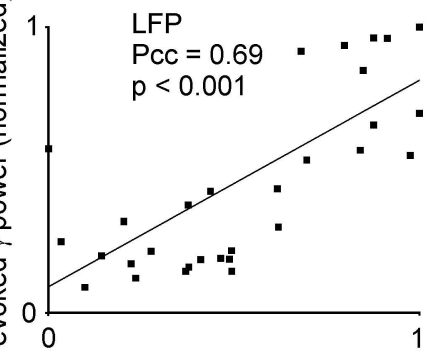

evoked TC amplitude (normalized) ketamine

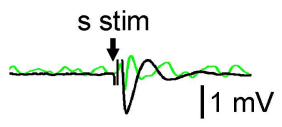

$\overline{100 \mathrm{~ms}}$

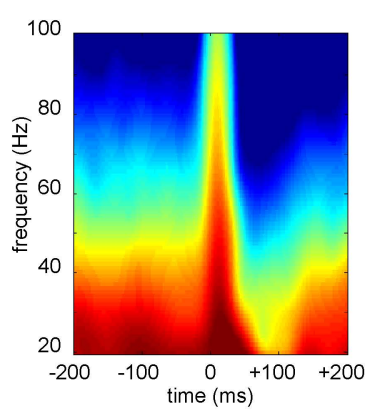

$10 * \log _{10}\left(\mu V^{2}\right)$

C
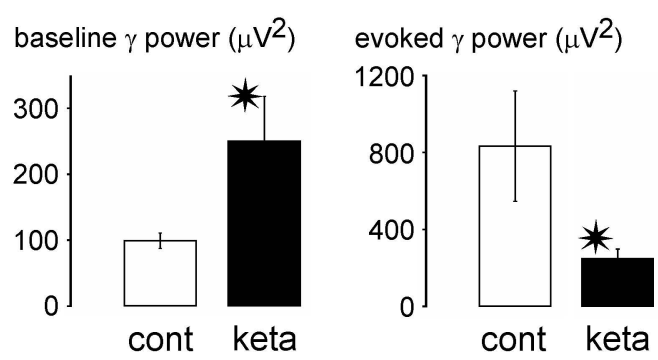
A

C
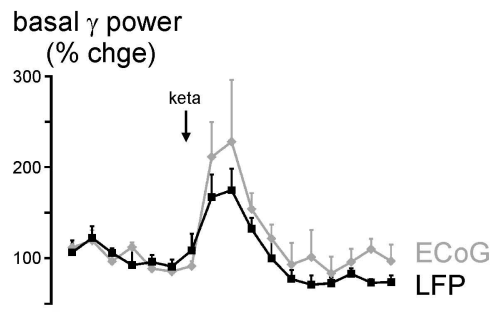

response amplitude (\% chge)

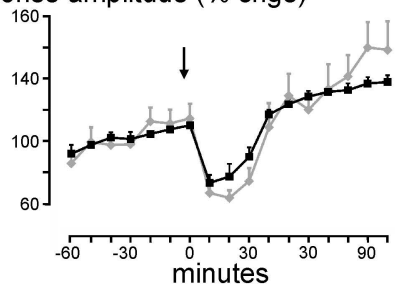

E

LFP amplitude

$160{ }^{(\% \text { chge })}$

120

1

100

80

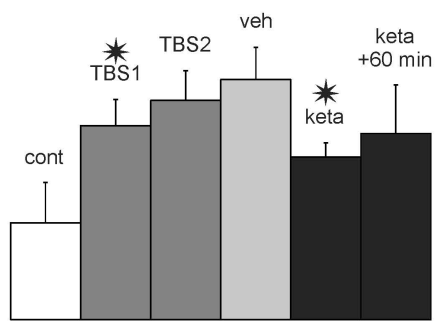

LFP amplitude

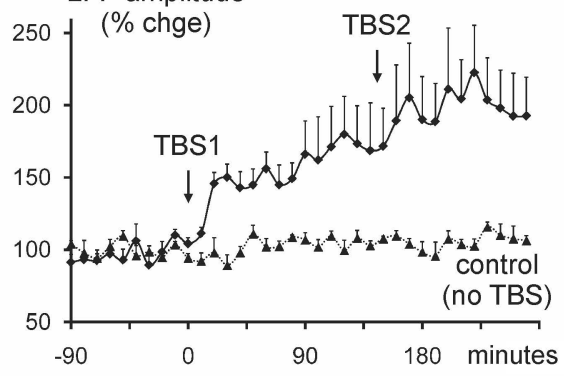

D

basal $\gamma$ power

(\% chge)

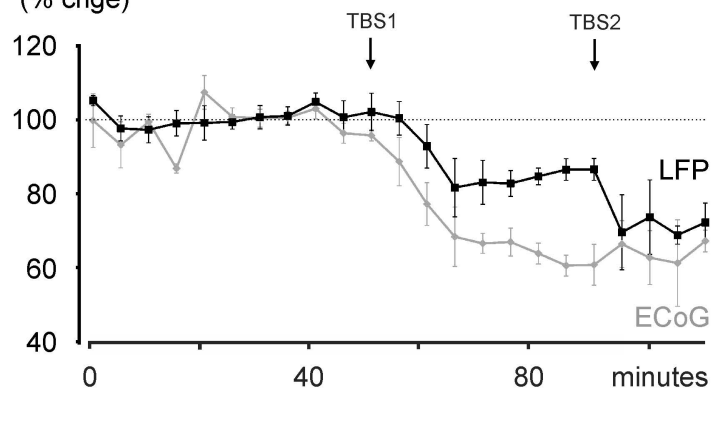

F LFP amplitude

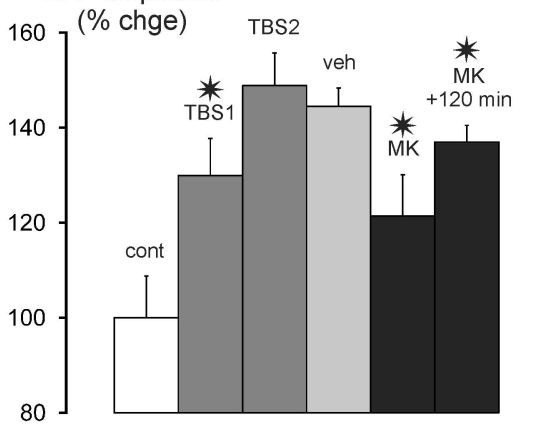


sensory

response basal

$\gamma$ oscillations

LTP

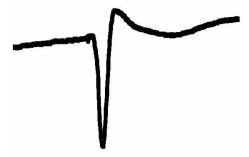

\section{WMW}

thalamic

hfDBS

\section{normal}

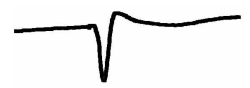

WWW

pathological

ketamine

\section{thalamic hfDBS?}
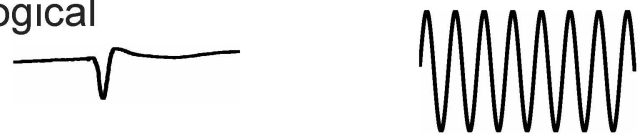